\title{
Comprimento de Desenvolvimento de Escoamentos Poiseuille Newtoniano com Escorregamento
}

\author{
Caroline Viezel ${ }^{1}$ \\ Instituto de Ciências Matemáticas e de Computação, ICMC - USP, São Carlos, SP \\ Gilcilene Sanchez de Paulo \\ Departamento de Matemática e Computação, FCT - UNESP, Presidente Prudente, SP
}

Resumo. Neste trabalho apresentam-se os resultados obtidos pela análise numérica do comprimento de desenvolvimento $\mathcal{L}$ em escoamentos Poiseuille Newtonianos com condição slip na parede. Os resultados numéricos são decorrentes das simulações na plataforma Freeflow-2D alterada para resolver problemas confinados com condição slip na parede.

Palavras-chave. Escoamentos Poiseuille, Comprimento de desenvolvimento, Condição slip, Simulação computacional.

\section{Introdução}

Escoamentos laminares de Poiseuille, caracterizados como escoamentos totalmente desenvolvidos, são clássicos em mecânica dos fluidos computacional principalmente na verificação de códigos e novas metodologias numéricas. Neste tipo de escoamento é necessário uma certa distância axial da entrada do canal para se obter a velocidade parabólica desenvolvida, caso seja imposto um perfil de velocidade uniforme nesse contorno. Sendo assim, define-se comprimento de entrada ou comprimento de desenvolvimento a distância axial da entrada de um canal ou tubo até o ponto onde o escoamento encontra-se em regime totalmente desenvolvido.

Na literatura, há diversos estudos sobre o comprimento de desenvolvimento em escoamentos Newtonianos levando em consideração a condição no-slip na parede (ver [3]). Em contribuição a ampliação dessas pesquisas, Ferrás et al. [4] apresentaram uma análise do comprimento de desenvolvimento considerando a condição slip na parede.

Neste trabalho, serão apresentados resultados numéricos paralelos aos obtidos por Ferrás et al. em [4], com o objetivo inicial de verificar a metodologia desenvolvida em [9], a qual poderá ser estendida, posteriormente, para resolver problemas mais complexos de interesse industrial. Desta forma, considerou-se um escoamento Newtoniano planar em um canal, como mostrado na Figura 1, com perfil de entrada reto $U=1 \mathrm{~m} / \mathrm{s}$. Assim como

\footnotetext{
${ }^{1}$ cviezel@usp.br

2 gilcilene@fct.unesp.br
} 
em [4], o comprimento $\mathcal{L}$ foi determinado sobre o eixo de simetria $y=0$, a partir da entrada do canal até a posição axial onde a velocidade numérica atingisse $99 \%$ da velocidade máxima $u_{\max }$.

\section{Formulação Matemática}

As equações que regem escoamentos Newtoniano laminares incompressíveis e isotérmicos são as equações da continuidade e momento,

$$
\begin{aligned}
\nabla \cdot \mathbf{u} & =0, \\
\frac{\partial \mathbf{u}}{\partial t}+\nabla \cdot\left(\mathbf{u} \mathbf{u}^{\top}\right) & =-\nabla p+\frac{1}{R e} \nabla^{2} \mathbf{u},
\end{aligned}
$$

sendo u o campo vetorial de velocidade, a variável escalar $p$ é a pressão e $t$ é a variável temporal. Neste trabalho, o número de Reynolds é definido pela largura do canal $L$, a velocidade média do escoamento $U$, a viscosidade $\eta_{s}$ do fluido Newtoniano e a densidade $\rho$ do fluido que em escoamentos incompressíveis é constante, $R e=\frac{\rho U L}{\eta_{s}}$.

As Equações (1) e (2) são escritas em coordenadas Cartesianas bidimensionais, adotandose a seguinte representação para o campo de velocidades, $\mathbf{u}=[u(x, y, t) v(x, y, t)]^{\top}$. Para resolvê-las considera-se a condição inicial $\mathbf{u}=\mathbf{u}\left(x, y, t_{0}\right)=\mathbf{0}$ para todo $(x, y)$ no interior do canal e $t_{0}=0$ o tempo inicial, e impõe-se as condições de contorno descritas a seguir.

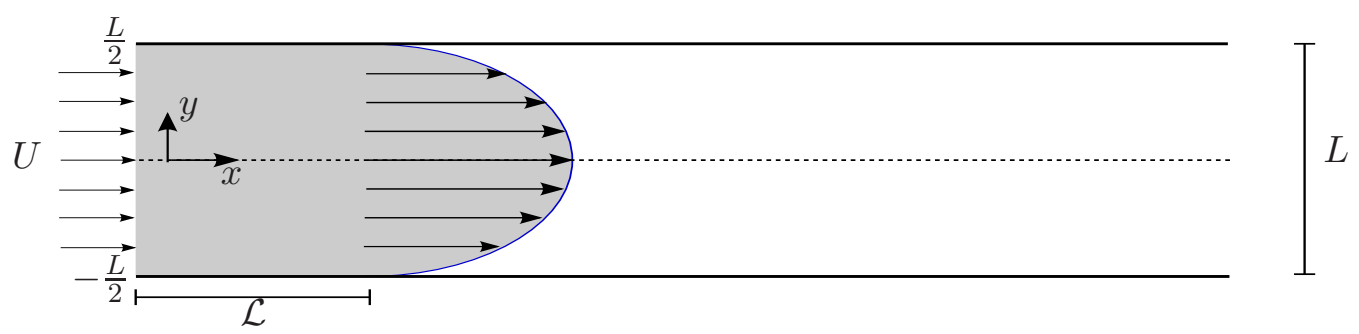

Figura 1: Esquema de domínio do problema.

$\mathrm{Na}$ entrada do canal, fronteira denominada por inflow ou injetor, considera-se as componentes de velocidades prescritas por $u=U$ e $v=0$, enquanto que na saída do canal (outflow ou ejetor) admite-se a condição de Neumann homogênea na direção normal a fronteira, direção $x: \frac{\partial u}{\partial x}=0$ e $\frac{\partial v}{\partial x}=0$. A fronteira rígida, wall ou parede, será impermeável, $v=0$, e escorregadia. Para representar o escorregamento na parede será adotada a lei de escorregamento Navier-linear [5,7], cuja lei é uma relação linear entre a velocidade de escorregamento e a tensão de cisalhamento na parede,

$$
u_{\text {wall }}=\left.\mp k_{n l} \frac{1}{R e} \frac{\partial u}{\partial y}\right|_{\text {wall }},
$$

onde o parâmetro $k_{n l}$, denominado constante de fricção, regula a intensidade do escorregamento do fluido na parede e os sinais $(\mp)$ resultam do fato de que as derivadas possuem 
sinais opostos nas paredes, ou seja, $\frac{\partial u}{\partial y}<0$ na parede superior e $\frac{\partial u}{\partial y}>0$ na parede inferior. Outro detalhe da Equação (3) é que sua forma adimensional está baseada na definição $k_{n l}=\rho U \overline{k_{n l}}$, onde a constante de fricção $\overline{k_{n l}}$ possui unidade de medida $\mathrm{m}^{2} \mathrm{~s} / \mathrm{Kg}$.

\section{Breve descrição do método numérico}

As Equações (1) e (2) acompanhadas das condições iniciais e de contorno, que foram descritas na seção 2 não são resolvidas diretamente. Por se tratar de escoamentos incompressíveis, é possível desacoplar velocidade e pressão nas Equações de Navier-Stokes com o auxílio de uma função potencial $\psi$ oriunda do Teorema da Decomposição de HelmholtzHodge. Essa segregação é conhecida como método de projeção ( [2]). Assim, a metodologia numérica consiste em resolver as Equações (4)-(7) descritas a seguir no algoritmo computacional.

A plataforma de simulação numérica de escoamentos incompressíveis Freeflow-2D resolve as Equações de Navier-Stokes da maneira descrita acima ( [6]) com a ressalva de utilizar apenas a condição de não-escorregamento na parede. Essa plataforma foi a base para a implementação da nova metodologia numérica, que inseriu um tratamento numérico para a condição de contorno de escorregamento na parede e suas implicações no novo sistema de equações. Desta forma, o novo método numérico foi desenvolvido pela técnica de diferenças finitas em uma malha deslocada adotando-se como base a formulação semiimplícita para o tratamento da velocidade [8]. Com exceção dos termos convectivos, que são discretizados pela técnica de alta ordem CUBISTA [1], as demais derivadas espaciais são discretizadas por diferenças centrais, salvo se os pontos não estiverem próximos aos contornos, onde passam a ser adotadas as diferenças regressivas ou progressivas.

Resumidamente o algoritmo computacional constitui-se dos seguintes passos. Admitese conhecida no instante $t_{n}: \mathbf{u}^{n}=\left[\begin{array}{ll}u^{n} & v^{n}\end{array}\right]^{\top}$ e $p^{n}$.

Passo 1: Prescreve-se $\widetilde{p}^{n+1}$ fazendo, $\widetilde{p}^{n+1}=p^{n}$.

Passo 2: Determina-se o campo de velocidade intermediário,

$$
\frac{\widetilde{\mathbf{u}}^{n+1}}{\delta t}-\frac{1}{R e} \nabla^{2} \widetilde{\mathbf{u}}^{n+1}=\frac{\widetilde{\mathbf{u}}^{n}}{\delta t}-\nabla \cdot\left(\mathbf{u} \mathbf{u}^{\top}\right)^{n}-\nabla \widetilde{p}^{n+1},
$$

fazendo $\widetilde{\mathbf{u}}^{n}=\mathbf{u}^{n}$ em todo o domínio e em todos os contornos impõe-se $\widetilde{\mathbf{u}}^{n+1}=\mathbf{u}^{n}$. De acordo com o texto, no contorno rígido pode-se escrever $\widetilde{\mathbf{u}}^{n+1}=\mathbf{u}_{\text {wall }}^{n}$.

Passo 3: Calcula-se $\psi^{n+1}$ via a equação de Poisson,

$$
\nabla^{2} \psi^{n+1}=\nabla \cdot \widetilde{\mathbf{u}}^{n+1},
$$

sendo $\frac{\partial \psi}{\partial \vec{n}}=0$ a condição de contorno nas paredes e inflow, e $\psi=0$ no outflow.

Passo 4: Atualiza-se o campo de velocidade no interior do domínio,

Passo 5: Calcula-se o novo campo de pressão,

$$
\mathbf{u}^{n+1}=\widetilde{\mathbf{u}}^{n+1}-\nabla \psi^{n+1} \text {. }
$$

$$
p^{n+1}=\widetilde{p}^{n+1}+\frac{\psi^{n+1}}{\delta t}-\frac{1}{R e} \nabla^{2} \psi^{n+1} .
$$

Passo 6: Atualizam-se as componentes da velocidade nos contornos. 


\subsection{Implementação numérica da condição slip na malha deslocada}

Considere a configuração de células adjacentes a parede como na Figura 2. A interface entre a célula exterior, fantasma, $[\mathbf{B}]$ e a célula de interior, com fluido, $[\mathbf{F}]$ representa a parede. A malha é deslocada, logo, a condição de impermeabilidade $v=0$ é imposta

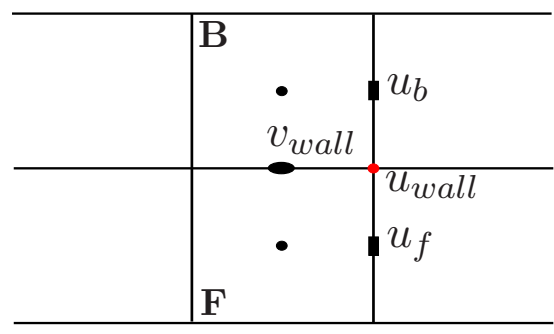

Figura 2: Representação da posição dos pontos envolvidos na discretização da velocidade na parede.

diretamente sobre a parede, na posição $v_{\text {wall }}$. Na malha deslocada a componente $u$ da velocidade fica armazenada no ponto médio das faces verticais das células. Ao discretizar os termos convectivos pelo método upwind na posição de $u_{f}$, o valor da velocidade na posição $u_{b}$ pode ser requerido. Neste trabalho, adotou-se a seguinte,

$$
u_{b}^{n}=2 u_{w a l l}^{n}-u_{f}^{n},
$$

sendo o valor de $u_{\text {wall }}$ fornecido pela condição slip, ou seja, pela Equação (3) discretizada sobre a parede, no vértice inferior direito da célula $[\mathbf{B}]$, da seguinte forma:

$$
u_{w a l l}=-\left.k_{n l} \frac{1}{R e} \frac{\partial u}{\partial y}\right|_{w a l l}=-k_{n l} \frac{1}{R e} \frac{u_{b}-u_{f}}{\delta y}=-\frac{2 k_{n l}}{R e} \frac{u_{w a l l}-u_{f}}{\delta y}
$$

$\log \mathrm{O}$

$$
u_{\text {wall }}^{n}=\frac{\frac{k_{n l}}{R e}}{\left(\frac{k_{n l}}{R e}+\frac{\delta y}{2}\right)} u_{f}^{n} .
$$

Portanto, calcula-se primeiramente $u_{\text {wall }}$ da Equação (10) e posteriormente atualiza-se o valor de $u_{b}$ pela Equação (8).

Uma descrição mais detalhada das estratégias numéricas adotadas para resolver escoamentos confinados incompressíveis Newtonianos e, inclusive, viscoelásticos com escorregamento será encontrada em [9].

\section{Resultados}

Nesta seção será analisado o comprimento $\mathcal{L}$ de desenvolvimento de um escoamento incompressível em um canal (ver Figura 1). O comprimento $\mathcal{L}$ é determinado sobre o eixo de simetria $y=0$, a partir da entrada do canal até a posição axial em que a velocidade 
numérica atinja $99 \%$ da velocidade máxima $u_{\max }$. Considera-se os seguintes dados: $L=$ $1 \mathrm{~m}$, comprimento do canal é $10 L, U=1 \mathrm{~m} / \mathrm{s}$. A malha computacional adotada possui $410 \times 41$ células, ou seja, espaçamento $\delta x=\delta y=0.02439$. O passo de tempo adotado foi $\delta t=1.6 \times 10^{-3}$.

Na Tabela 1 encontram-se os valores de $\mathcal{L}$ obtidos pela metodologia descrita neste trabalho e o erro relativo da velocidade com base na solução analítica (ver Apêndice A, Equação (11)). Além disso, apresenta-se na Tabela 1 o comprimento $\mathcal{C}$ obtido por Ferrás et al. em [4].

Tabela 1: Comprimento de desenvolvimento e erro relativo da velocidade $u$. Resultados $\mathcal{L}$ foram obtidos por este trabalho e $\mathcal{C}$ foram obtidos por Ferrás et al. [4].

\begin{tabular}{|c|c|c|c|c|}
\hline$R e$ & $k_{n l}$ & $\mathcal{L}$ & $\mathcal{C}$ & $\left\|u_{\text {Ext }}-u_{\text {Num }}\right\|_{2} /\left\|u_{\text {Ext }}\right\|_{2}$ \\
\hline \hline \multirow{5}{*}{0.001} & $10^{-7}$ & 0.6341 & 0.6284 & $5.547989 \times 10^{-4}$ \\
\cline { 2 - 5 } & $10^{-6}$ & 0.6341 & 0.6293 & $5.500617 \times 10^{-4}$ \\
\cline { 2 - 5 } & $10^{-5}$ & 0.6341 & 0.6377 & $5.061709 \times 10^{-4}$ \\
\cline { 2 - 5 } & $10^{-4}$ & 0.6829 & 0.6816 & $2.682089 \times 10^{-4}$ \\
\cline { 2 - 5 } & $10^{-3}$ & 0.5610 & 0.5560 & $4.376375 \times 10^{-5}$ \\
\hline \hline \multirow{5}{*}{1.0} & $10^{-4}$ & 0.6341 & 0.6411 & $5.547983 \times 10^{-4}$ \\
\cline { 2 - 5 } & $10^{-3}$ & 0.6341 & 0.642 & $5.500986 \times 10^{-4}$ \\
\cline { 2 - 5 } & $10^{-2}$ & 0.6341 & 0.6509 & $5.061717 \times 10^{-4}$ \\
\cline { 2 - 5 } & $10^{-1}$ & 0.7073 & 0.7 & $2.682146 \times 10^{-4}$ \\
\cline { 2 - 5 } & 1.0 & 0.5610 & 0.5779 & $4.368362 \times 10^{-5}$ \\
\hline \hline \multirow{5}{*}{10.0} & $10^{-3}$ & 0.8049 & 0.8144 & $5.548178 \times 10^{-4}$ \\
\cline { 2 - 5 } & $10^{-2}$ & 0.8049 & 0.8159 & $5.500586 \times 10^{-4}$ \\
\cline { 2 - 5 } & $10^{-1}$ & 0.8293 & 0.831 & $5.061765 \times 10^{-4}$ \\
\cline { 2 - 5 } & 1.0 & 0.9512 & 0.9388 & $2.681705 \times 10^{-4}$ \\
\cline { 2 - 5 } & 10.0 & 0.8293 & 0.8441 & $4.377037 \times 10^{-5}$ \\
\hline
\end{tabular}

Observando os dados da Tabela 1 e a Figura 3 verifica-se que o comprimento de desenvolvimento possui uma relação não linear com o coeficiente de friç̧ão, assim como Ferrás et al. já tinham observado em [4] (ver coluna $\mathcal{C}$, na Tabela 1) com uma metodologia numérica diferente da apresentada no presente trabalho.

Para valores de $k_{n l}$ suficientemente grandes, por exemplo, o caso $k_{n l}=10^{-2}$ e $R e=$ 0.001 , percebe-se que a partir da entrada do canal $(x=0)$, a velocidade já ultrapassa $99 \%$ da velocidade máxima. Isso fica bem mais evidente observando a Figura 4, que apresenta o comportamento da velocidade sobre o eixo de simetria $y=0$. Nesse gráfico a variável $u$ é normalizada pela velocidade máxima $u_{\max }$ obtida através da solução analítica. A reta indica quando $u / u_{\max }$ é exatamente igual a $99 \%$. 

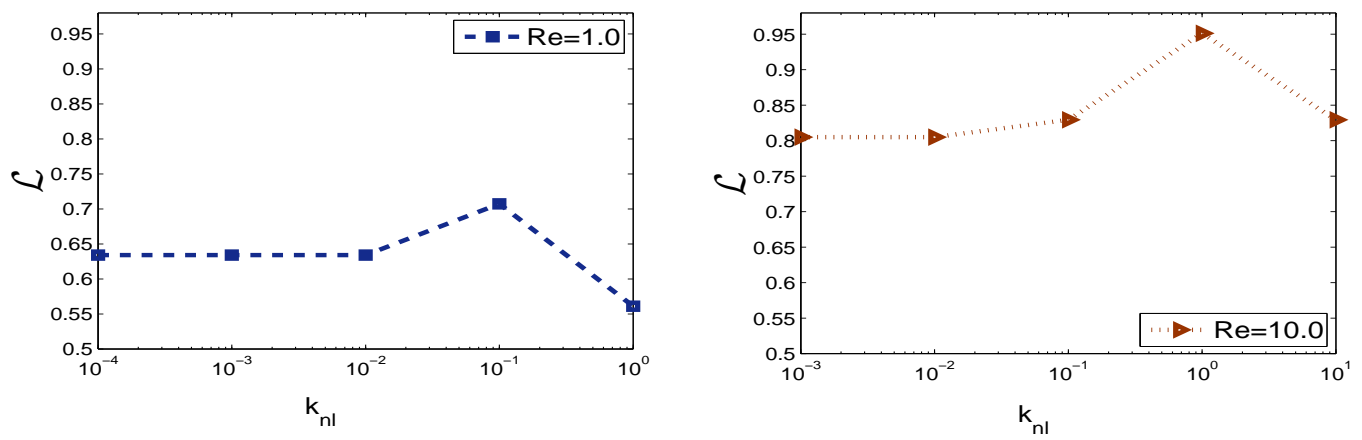

Figura 3: Comprimento de desenvolvimento do escoamento de fluido Newtoniano versus a constante de fricção adimensional $k_{n l}$.

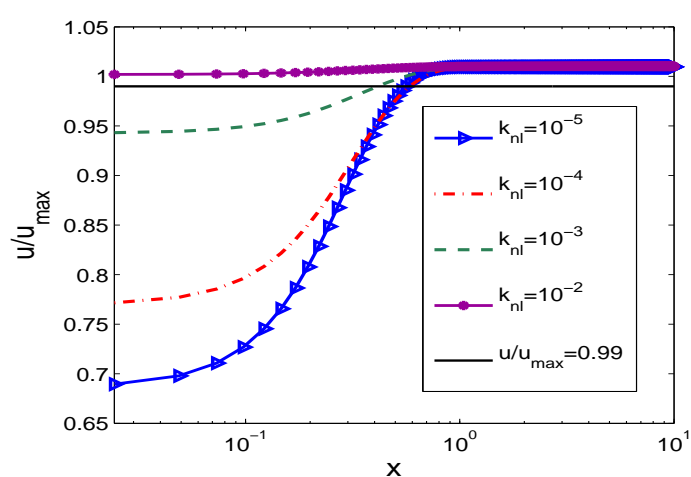

(a)

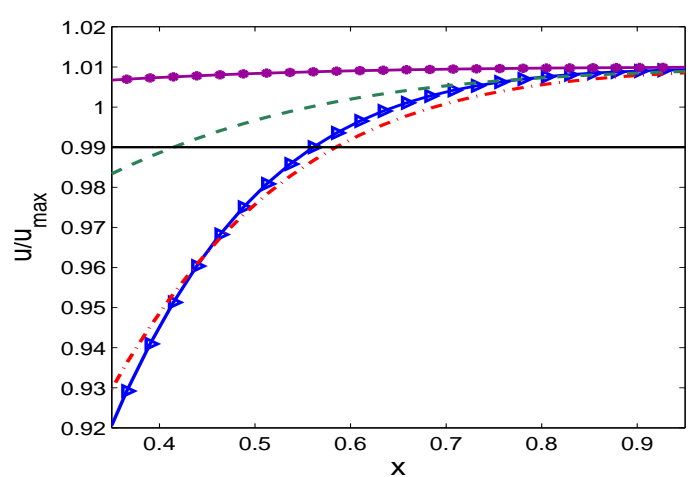

(b)

Figura 4: Velocidade sobre o eixo de simetria normalizada pela velocidade $u_{\max }$ analítica para $R e=0.001$ com diferentes coeficientes de fricção $\left(k_{n l}\right)$. (b) ampliação de $(a), y \in[0.35,0.95]$.

\section{Conclusões}

A metodologia numérica apresentada para resolver escoamentos incompressíveis sob a hipótese de escorregamento na parede, baseada na técnica de diferenças finitas sobre uma malha deslocada mostrou-se eficiente para resolver estes escoamentos com escorregamento em geometria simples. Os resultados numéricos do comprimento de desenvolvimento estão de acordo com os já apresentados na literatura, ou seja, verificou-se que o comprimento de desenvolvimento possui uma relação não linear com o coeficiente de fricção para os valores de Reynolds considerados. A presente metodologia pode ser aplicada, com algumas estensões na implementação, para escoamentos com escorregamento em geometrias mais complexas, de interesse industrial. 


\section{Agradecimentos}

As autoras agradecem a FAPESP (Processo 2013/25620-1) e a CAPES pelo apoio financeiro.

\section{Referências}

[1] M.A. Alves, F.T. Pinho, and P.J. Oliveira. Study of steady pipe and channel flows of a single-mode phan-thien tanner fluid. Journal of Non-Newtonian Fluid Mechanics, 101:55-76, 2001. DOI: 10.1016/S0377-0257(01)00159-8.

[2] A. Chorin. A numerical method for solving incompressible viscous flow problems. $J$. Comp. Physics, 2:12-26, 1967. DOI: 10.1006/jcph.1997.5716.

[3] F. Durst, S. Ray, B. Ünsal, and O. Bayoumi. The development lengths of laminar pipe and channel flows. Journal of Fluids Engineering, 127:1154-1160, 2005. DOI: 10.1115/1.2063088.

[4] L.L. Ferrás, A.M. Afonso, M.A. Alves, J.M. Nóbrega, and F.T. Pinho. Development length in planar channel flows of newtonian fluids under the influence of wall slip. Journal of Fluids Engineering, 134(10):104503, 2012. DOI: 10.1115/1.4007383.

[5] L.L. Ferrás. Theoretical and numerical studies of slip flows. Tese de Doutorado, Universidade do Minho, 2012.

[6] S. McKee; M.F. Tome; V.G. Ferreira; J.A. Cuminato; A. Castelo; F.S. Sousa; N. Mangiavacchi. The mac method. Computers and Fluids, 37:907-930, 2008.

[7] C.L.M.H. Navier. Mémoire sur les lois du mouvement des fluides. mémoires de l'académie royale des sciences de l'institut de france. Sciences de L'Institut de France, VI:389-440, 1823.

[8] C.M. Oishi. Análise e implementação de métodos implícitos e de projeção para escoamentos com superfície livre. Tese de Doutorado, ICMC/USP, 2008.

[9] C. Viezel. Estudos teóricos e numéricos de escoamentos com escorregamento. Dissertação de Mestrado, FCT/UNESP, 2015.

\section{A Solução Analítica}

A solução analítica para escoamentos incompressíveis e isotérmicos totalmente desenvolvidos em um canal sob a condição de contorno Navier-linear (3) é dada pela expressão,

$$
u(y)=-\frac{p_{x} R e}{8}\left[1-4 y^{2}\right]-k_{n l}\left[\frac{1}{2} p_{x}\right],
$$

sendo $p_{x}$ o gradiente de pressão constante. Maiores detalhes sobre a obtenção desta solução analítica e, também, para o caso de fluidos viscoelásticos PTT são encontrados em [9]. 